\title{
Cognition-aware Cognate Detection
}

\author{
Diptesh Kanojia ${ }^{\dagger, \star, \star}$, Prashant Sharma $^{\diamond}$, Sayali Ghodekar ${ }^{\ddagger}$, \\ Pushpak Bhattacharyya $^{\dagger}$, Gholamreza Haffari ${ }^{\star}$, and Malhar Kulkarni ${ }^{\dagger}$ \\ ${ }^{\dagger}$ IIT Bombay, India, ${ }^{\diamond}$ Hitachi CRL, Japan, ${ }^{\ddagger}$ RingCentral, India \\ *IITB-Monash Research Academy, India \\ ${ }^{\star}$ Monash University, Australia \\ $\dagger\{$ diptesh,pb, malhar\}@iitb.ac.in, ^prashaantsharmaa@gmail.com \\ ${ }^{\ddagger}$ sayalighodekar26@gmail.com, *gholamreza.haffari@monash.edu
}

\begin{abstract}
Automatic detection of cognates helps downstream NLP tasks of Machine Translation, Cross-lingual Information Retrieval, Computational Phylogenetics and Cross-lingual Named Entity Recognition. Previous approaches for the task of cognate detection use orthographic, phonetic and semantic similarity based features sets. In this paper, we propose a novel method for enriching the feature sets, with cognitive features extracted from human readers' gaze behaviour. We collect gaze behaviour data for a small sample of cognates and show that extracted cognitive features help the task of cognate detection. However, gaze data collection and annotation is a costly task. We use the collected gaze behaviour data to predict cognitive features for a larger sample and show that predicted cognitive features, also, significantly improve the task performance. We report improvements of $10 \%$ with the collected gaze features, and $12 \%$ using the predicted gaze features, over the previously proposed approaches. Furthermore, we release the collected gaze behaviour data along with our code and cross-lingual models.
\end{abstract}

\section{Introduction}

Cognates are word pairs, across languages, which have a common etymological origin. For example, the French and English word pair, Liberté - Liberty, reveals itself to be a cognate through orthographic similarity. The task of automatic cognate detection across languages ${ }^{1}$ requires one to detect word pairs which are etymologically related, and carry the same meaning. Such word pairs share a formal and/or semantic affinity and facilitate the second language acquisition process, particularly between related languages. Although they can accelerate vocabulary acquisition, language learners also need

\footnotetext{
${ }^{1}$ Cognates can also exist in the same language. Such word pairs/sets are commonly referred to as doublets.
}

to be aware of false friends and partial cognates, at times, leading to unrelated semantic coupling. For example, "gift" in German means "poison", which is a known example of a False Friend pair. For an example of a partial cognate, the word "police" can translate to "police", "policy" or "font", in French, depending on the context in which it was used.

Manual detection of such cognate sets requires a human expert with a good linguistic background in multiple languages. Moreover, manual annotation of cognate sets is a costly task in terms of time and human effort. Automatic Cognate Detection (ACD) is a well-known task, which has been explored for a range of languages using different methods; and has shown to help Cross-lingual Information Retrieval (Meng et al., 2001), Machine Translation (MT) (Al-Onaizan et al., 1999), and Computational Phylogenetics (Rama et al., 2018). In the traditional approaches to automatic cognate detection, words with similar meanings or forms are used as probable cognates (Jäger et al., 2017; Rama, 2016; Kondrak, 2001). From such pairs, the ones that exhibit a high phonological, lexical and/or semantic similarity, are analyzed in order to find true cognates. Merlo and Andueza Rodriguez (2019) perform an investigation to evaluate the use of crosslingual embeddings and show that these models inherit a lexical structure matching the bilingual lexicon. This study establishes that cross-lingual models can provide an effective similarity score compared to their monolingual counterparts, for both cognates and false friends. However, they do not evaluate machine learning (ML) approaches that distinguish between cognates and false friends. The absence of such an evaluation motivates us to use cross-lingual similarity scores with ML algorithms. Our work ${ }^{2}$ reports whether these scores can provide an adequate distinction or not.

\footnotetext{
${ }^{2}$ Dataset and Code
} 


\begin{tabular}{ccccc}
\hline & Hindi (Hi) & Marathi (Mr) & Hindi Meaning & Marathi Meaning \\
\hline $\begin{array}{c}\text { Cognate } \\
\text { False Friend }\end{array}$ & ank & ank & Number & Number \\
shikshA & shikshA & Education & Punishment \\
\hline
\end{tabular}

Table 1: An example each of a cognate and a false friend pair from Indian languages, Hindi and Marathi.

Inspired from their work, we investigate the use of cross-lingual word embeddings and cognitive features to distinguish between cognates and false friends, for the Indian language pair of Hindi and Marathi. Cognitively inspired features have shown to improve various NLP tasks (Mishra et al., 2018a). However, most of their work involves collecting the gaze behaviour data first on a large sample, and then splitting the data into training and testing data, before performing their experiments. While their work does show significant improvements over baseline approaches, across multiple NLP tasks, collecting gaze behaviour over a large $\operatorname{cog}$ nate dataset can be costly, both in terms of time and money. Our approach tries to reduce annotation cost by predicting gaze behaviour data for a large sample based on the smaller sample of collected gaze data. Our investigations use three recently proposed cross-lingual word embeddings based approaches to generate features for the task of cognate detection. We also generate cognitive features from participants' gaze behaviour data and show that gaze behaviour helps the task of cognate detection. Additionally, we use the collected gaze behaviour data and predict gaze-based features for a much larger sample. We believe that using gaze features will be more applicable only if gaze features can be predicted for unseen samples. We believe that collection of gaze data cannot be performed in all the scenarios and hence hypothesize that predicting such data if it helps improve the task of cognate detection, should be a viable solution.

\section{Motivation}

Consider a scenario where an NLP task comes across the false friend pair in Table 1. Orthographic similarity or even phonetic similarity-based techniques will fail to detect the difference between the Hindi meaning of the word "shikhshA" and its Marathi counterpart. Here, semantic approaches should detect the distinction in meaning, but monolingual embeddings are trained using a large corpus from the same language. In such cases, it becomes imperative that a cross-lingual word embeddings model be utilized. However, Indian languages are known to be low-resource languages compared to English or even many European languages like French, Italian, German etc. Acquiring additional clean data for training cross-lingual models is, yet again, a painful task. In such a scenario then, we ask ourselves,

"Can cognitive features be used to help the task of Cognate Detection?"

furthermore,

"Using gaze features collected on a small set of data points, can we predict the same features on a larger set of data points to alleviate the need for collecting gaze data?"

With this work, we try to answer both the questions stated above and present the rest of the paper as follows. We discuss the current literature on the cognate detection task and cognitive NLP in Section 2. Section 3 discusses the dataset acquisition, including the description and analysis of our gaze behaviour data. We describe the feature sets used in section 4. Our approaches to the task of cognate detection are discussed in Section 5. The results of our work are discussed in Section 6. Finally, we conclude the study with possible future work in this direction in Section 7.

\section{Terminology}

An interest area (IA) is an area of the annotation screen to be processed by the human reader. In our experiments, it is an area where a word is shown to the reader. A fixation is an event where the reader focuses within an "interest area". A saccade is the movement of the eye from one fixation point to another. If the saccades move from an earlier IA to a later IA, such a saccade is called a progression. A regression is the saccade path when the reader moves back to a previous IA. We also use the terms reader and participant interchangeably.

\section{Related Work}

Current literature which uses gaze behaviour to solve downstream NLP tasks has been applied to the NLP tasks of sentiment analysis (Mishra et al., 2018a; Barrett et al., 2018; Long et al., 2019), sarcasm detection (Mishra et al., 2016), grammatical error detection (Barrett et al., 2018), hate speech detection (Barrett et al., 2018), named entity recognition (Hollenstein and Zhang, 2019), part- 
of-speech tagging (Barrett et al., 2016), sentence simplification (Klerke et al., 2016), and readability (González-Garduño and Søgaard, 2018; Singh et al., 2016). A comprehensive overview is provided by Mishra and Bhattacharyya (2018). The primary motivation of using cognitive features for NLP tasks is derived from the eye-mind hypothesis (Just and Carpenter, 1980), which establishes a direct correlation between a reader's comprehension of the text with the time taken to read the text. This hypothesis has initiated a large body of psycholinguistic research that shows a relationship between text processing and gaze behaviour. Yaneva et al. (2020) discuss the use of gaze features for the task of anaphora resolution. Their findings show that gaze data can substitute the classical text processing approaches along with the fact that human disambiguation process overlaps with the information carried in linguistic features. Rohanian (2017) use gaze data to automatically identify multiword expressions and observe that gaze features help improve the accuracy of the task when combined with traditional linguistic features used for the task.

Mathias et al. (2020) describe an approach to scoring essays in a multi-task learning framework automatically. Their approach relies on collecting gaze behaviour for essay reading for a small set of essays and then predicting the rest of the dataset's gaze behaviour in a multi-task learning setup. Similarly, Barrett et al. (2016) use token level averages of cognitive features at run time, to mitigate the need for these features at run time. Singh et al. (2016) and Long et al. (2019) predict gaze behaviour at the token-level as well. Mishra et al. (2018a), González-Garduño and Søgaard (2018), Barrett et al. (2018), and Klerke et al. (2016), use multi-task learning to solve the primary NLP task, where learning the gaze behaviour is an auxiliary task.

Orthographic/String similarity-based methods are often used as baseline methods for the cognate detection task, and the most commonly used method amongst them is the Edit distance-based similarity measure (Melamed, 1999; Mulloni and Pekar, 2006). Research in automatic cognate detection using various aspects involves the computation of similarity by decomposing phonetically transcribed words (Kondrak, 2000; Dellert, 2018), acoustic models (Mielke et al., 2012), clustering based on semantic equivalence (Hauer and Kondrak, 2011), and aligned segments of transcribed phonemes (List, 2012). Rama (2016) employs a Siamese convolutional neural network to learn the phonetic features jointly with language relatedness for cognate identification. Jäger et al. (2017) use SVM for phonetic alignment and perform cognate detection for various language families. Various works on orthographic cognate detection usually take alignment of substrings within classifiers like SVM (Ciobanu and Dinu, 2014, 2015) or HMM (Bhargava and Kondrak, 2009). Ciobanu and Dinu (2014) employ dynamic programming based methods for sequence alignment. Cognate facilitation in second language learners has previously been explored with the help of eye-tracking studies (Blumenfeld and Marian, 2005; Van Assche et al., 2009; Bosma and Nota, 2020) but the task of cognate detection has not been performed with gaze features obtained from the cognitive data in any of the previously available literature.

For the task of cognate detection, however, the use of cognitive features has not been established previously. The task of cognate detection is crosslingual, and a reader's cognitive load should vary while trying to comprehend the meaning of concepts, from different languages. Our work tries to exploit the difference noted in terms of time taken and eye-movement patterns in cognates $v s$ false friends, to generate additional features for the ACD task. Moreover, for Indian languages such as Marathi, where agglutination ${ }^{3}$ varies the word length, the task becomes tougher, computationally.

\section{Dataset Acquisition \& Analysis}

We pose the problem of cognate detection as a binary classification task in a supervised setting. We use a recently released challenging dataset (Kanojia et al., 2020) of cognates and false friends. We extract the Hindi-Marathi cognate and false friend pairs. The number of cognate and false friend pairs released by the paper cited above is 15726 , and 5826. We select an equal number of cognates at random to reduce this skew, thus producing a balanced dataset for the classification task. For any further experiments in our paper, we use this artificially class-balanced dataset of 5826 (cognates) + 5826 (false friends) data points. We also augment the complete dataset with context information from the IndoWordnet (Bhattacharyya, 2017). The con-

\footnotetext{
${ }^{3}$ Agglutination is a linguistic process pertaining to derivational morphology in which complex words are formed by stringing together morphemes without changing them in spelling or phonetics.
} 


\begin{tabular}{ccc}
\hline & Cognates (1) & False Friends (0) \\
\hline Kanojia et. al. (2020) & 15726 & 5826 \\
D1 & $\mathbf{5 8 2 6}$ & $\mathbf{5 8 2 6}$ \\
D2 & $\mathbf{1 0 0}$ & $\mathbf{1 0 0}$ \\
\hline
\end{tabular}

Table 2: Dataset Statistics for Cognate Detection Task

text information contains a gloss and an example sentence from the Wordnet data. The dataset released by Kanojia et al. (2020) contains the Synset IDs for each word pair, which helps us locate exact concept information from the dataset. We provide positive labels to cognates and negative labels to false friend pairs obtained from this data and construct what we call "D1".

We extract 100 pairs, at random, from each of the positive and negative labels for collecting gaze behaviour data, to construct what we call "D2". This data, extracted from D1, is used to collect gaze behaviour and annotation. Although we have gold labels for the data extracted, we ask the participants to annotate the data by asking them if the concepts shown on the screen mean the same. The annotation screen provides them with contextual clues obtained from Wordnet data on the screen, as shown in Figure 1. The complete dataset statistics are shown in Table 2.

\subsection{Gaze Data Collection and Annotation}

The task assigned to annotators was to read word pairs and the contextual clues provided on the screen, one pair at a time. The annotators were requested to label the pairs with a binary score indicating the similarity in meaning (i.e., positive/negative). It should be noted that the participants were not instructed to annotate whether a pair is a cognate or a false friend, to rule out the Priming Effect, (i.e., if the exact task (cognates vs false friends) is expected beforehand, processing cognate pairs will become relatively easier (SáchezCasas et al., 1992)). This ensures the ecological validity of our experiment in two ways: (1) The participant does not have any clue so that they can treat cognates with special attention (done by asking them to annotate based solely on meaning similarity) (2) Cognate pairs are mixed with false friend pairs and the participant does not have any prior knowledge about whether the next word pair would be a cognate or not. This also ensures that the participants pay attention to the task and do not just skim through the word-pair presented on the screen.

\begin{tabular}{cccccc}
\hline & $\mu \_$Pos & $\sigma \_$Pos & $\mu \_$Neg & $\sigma \_$Neg & $\mathrm{p}$ \\
\hline P1 & 9.720 & 17.867 & 8.677 & 4.281 & 0.028 \\
P2 & 8.596 & 10.526 & 7.619 & 13.794 & 0.049 \\
P3 & 7.770 & 6.664 & 7.044 & 3.900 & 0.027 \\
P4 & 9.686 & 17.729 & 8.664 & 4.306 & 0.031 \\
P5 & 8.861 & 8.611 & 8.099 & 5.246 & 0.042 \\
P6 & 7.854 & 6.286 & 7.184 & 3.442 & 0.033 \\
P7 & 8.564 & 5.499 & 7.918 & 3.540 & 0.033 \\
P8 & 8.018 & 5.955 & 7.340 & 3.742 & 0.031 \\
P9 & 9.720 & 17.867 & 8.703 & 4.305 & 0.028 \\
\hline
\end{tabular}

Table 3: T-test statistics for average fixation duration time per word for Positive labels (Cognates) and Negative labels (False Friends) for participants P1-P9.

It should be noted that all our participants are primarily Marathi speakers and have learnt Hindi at the school level. Hindi and Marathi are considered to be relatively closer languages due to their shared vocabulary and the geographical location of the demographic. All the participants speak, understand and write - both Hindi and Marathi.

The collection of gaze data is conducted by following the standard norms in eye-movement research (Holmqvist et al., 2011). While reading, an SR-Research Eyelink-1000 eye-tracker (monocular remote mode, sampling rate $500 \mathrm{~Hz}$ ) records several eye-movement parameters like fixations (a long stay of gaze) and saccade (quick jumping of gaze between two positions of rest) and pupil size. For this experiment, the default value of $4 \mathrm{~ms}$ was used for a gaze to be counted as fixation. We request a total of 15 participants to perform the annotation task, out of which only 11 participants could perform the data collection ${ }^{4}$.

Out of the 11 completed annotations, we discarded the data from 2 participants as their gaze behaviour was erratic (the fixations were too far away from the IAs). The participants are graduates with science and engineering background. They are bilingual speakers who know both Hindi and Marathi. Our participants were given a set of instructions beforehand and were advised to seek clarifications before they proceed. The instructions mention the nature of the task as discussed above, annotation input method, and the necessity of head movement minimization during the experiment.

\footnotetext{
${ }^{4}$ We could not perform gaze data collection with the remaining 4 participants due to the COVID-19 pandemic.
} 


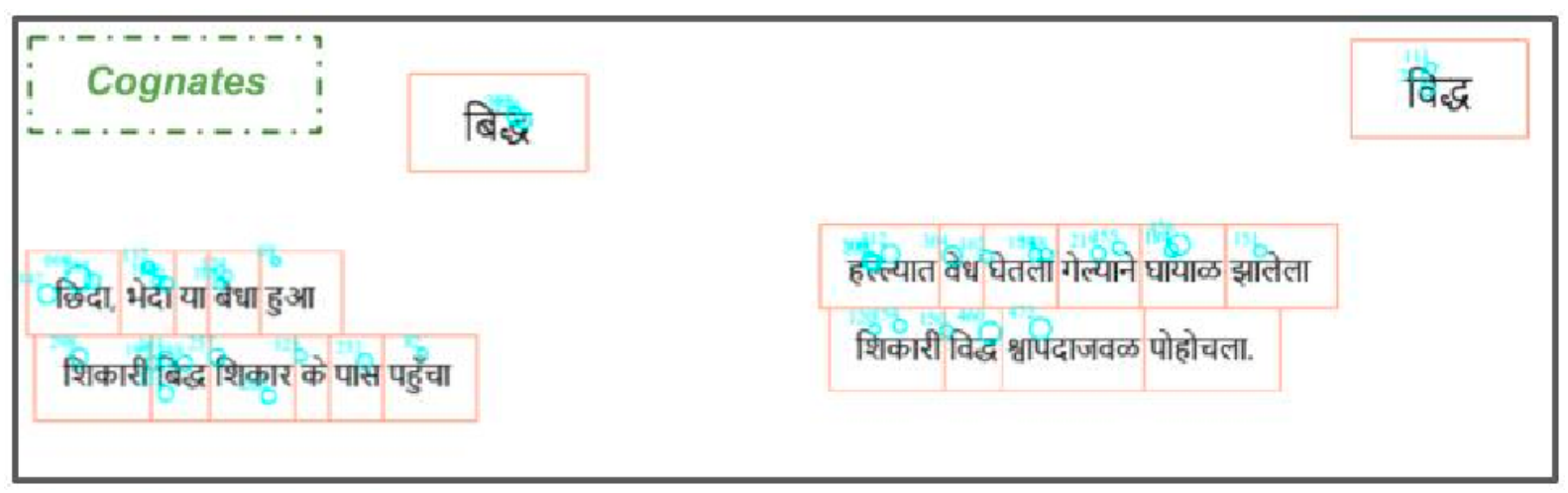

Figure 1: Screen capture showing collection of gaze features (via eye tracking) while displaying word pairs along with respective definitions and examples. The figure shows the cognate pair (BiDha - BiDha) where both mean "pierced" in the context of hunting. The figure also shown the glosses and example sentences provided to the annotator for this cognate pair in their respective languages (Hindi and Marathi)

\subsection{Gaze Behaviour Data Analysis}

The accuracy of similarity annotation by participants lies between $98 \%$ to $99.5 \%$ for individual annotators. Out of the 1800 annotations (9 annotators over 200 word-pairs), only 40 were predicted incorrectly. Annotation errors may be attributed to: (a) lack of patience/attention while reading, (b) issues related to word-pair comprehension, and (c) confusion/indecisiveness caused due to lack of contextual clues. In our analysis, we do not discard the data obtained from these incorrect annotations.

We observe distinct eye-movement patterns for cognate pairs in terms of fixation duration of the human readers. Our analysis shows that fixation duration normalized over word count is relatively larger for cognate pairs. For cognate pairs, we observe that average fixation duration amongst all participants is 1.3 times more than that of false friend pairs. To test the statistical significance, we conduct a two-tailed t-test (assuming unequal variance) to compare the average fixation duration per word for cognate and false friend pairs. The hypothesized mean difference is set to 0 , and the error tolerance limit $(\alpha)$ is set to 0.05 . The t-test analysis, presented in Table 3, shows that for all participants, a statistically significant difference exists between the average fixation duration per word for cognate pairs vs false friend pairs.

We believe this difference in average fixation duration is because the bilingual speakers who participated in the experiment can clearly distinguish between cognates and false friends and decide quickly in either case. The duration for which they fixate on either of the cases differs significantly for each participant. As per our observation, the participants take more time over cognate pairs to ensure similarity in meaning. Given their knowledge of both the languages and contextual clues, they were 'quickly' able to decide these word pairs did not mean the same. It should be noted that they were unaware of the 'cognate' or 'false friend' distinction concerning the experiment. They were simply asked to note the meaning of both the words given context, and annotate accordingly, as described in the paper.

\subsection{Cross-lingual Word Embeddings}

For this task, we use the cross-lingual word embedding models released by Kumar et al. (2020). The Hindi-Marathi cross-lingual models released with this paper are based on both MUSE (Conneau et al., 2017) and $\mathrm{XLM}^{5}$ (Lample and Conneau, 2019). Additionally, we build the cross-lingual word embeddings model for Hindi-Marathi using VecMap (Artetxe et al., 2017). The model uses monolingual corpora released by Kunchukuttan et al. (2020) and a bilingual dictionary ${ }^{6}$ required for the supervised method by Artetxe et al. (2017). These three cross-lingual models provide us with three feature sets for the task of cognate detection.

\section{Feature Sets for Cognate Detection}

In this section, we discuss the various features used for the task of cognate detection. It is to be noted that false friends are spelt similarly across languages but mean differently. Using false friends as data points with negative labels restricts us to the use of semantic similarity based features,

\footnotetext{
${ }^{5}$ Word representations extracted from the last layer of the contextual XLM model.

${ }^{6}$ Bilingual Lexicon
} 
as orthographic or phonetic similarity-based measures would fail to detect sufficient distinction between them. Hence, we use the features proposed by Rama (2016) and Kanojia et al. (2019a) as baseline features for a comparative evaluation.

\subsection{Phonetic Features}

The IndicNLP Library provides phonetic features based vector for each character in various Indian language scripts. We utilize this library to compute a feature vector for each word by computing an average over character vectors. We compute vectors for both words in the candidate cognate pairs $\left(P V_{S}\right.$ and $\left.P V_{T}\right)$ and also compute contextual vectors $\left(P C V_{S}\right.$ and $\left.P C V_{T}\right)$ by averaging the vectors for all the contextual clues, generating a total of four vectors. We use these vectors as features for computing the baseline scores using the Siamese Convolutional Neural Network architecture proposed by Rama (2016).

\subsection{Weighted Lexical Similarity (WLS)}

The Normalized Edit Distance (NED) approach computes the edit distance (Nerbonne and Heeringa, 1997) for all word pairs in our dataset. A combination of NED with q-gram distance (Shannon, 1948) for a better similarity score. The q-grams ('n-grams') are simply substrings of length q. This distance measure has been applied previously for various spelling correction approaches (Owolabi and McGregor, 1988; Kohonen, 1978). Kanojia et al. (2019b) proposed Weighted Lexical Similarity (WLS) and we use it with the character-based Recurrent Neural Network architecture proposed by them to compute another set of baseline scores.

\subsection{Cross-lingual Vectors \& Similarity}

As discussed above, we use the pre-trained crosslingual embedding models for generating feature vectors for MUSE and XLM based approaches. These models are generated by aligning two disjoint monolingual vector spaces through linear transformations, using a small bilingual dictionary for supervision (Doval et al., 2018; Artetxe et al., 2017). Additionally, the cross-lingual embeddings model trained using Artetxe et al. (2017)'s approach provides us with the third set of feature vectors.

We use these models to obtain vectors for wordpairs $\left(W V_{S}\right.$ and $\left.W V_{T}\right)$ and averaged context vectors $\left(C V_{S}\right.$ and $\left.C V_{T}\right)$ from the contextual clues, to create three different feature sets. We obtain vectors for each candidate pair and their context using all the three cross-lingual methodologies. The use of cross-lingual models has been proposed for differentiating between cognates and false friends by Merlo and Andueza Rodriguez (2019), but evaluation with the cognate detection task had not been performed. We perform this evaluation on our datasets $(\mathrm{D} 1, \mathrm{D} 2$ and $\mathrm{D} 1+\mathrm{D} 2)$ using various classification methods and discuss them later.

\subsection{Cognitive Features from Gaze Data}

Gaze behaviour of participants, characterized by fixations, forward saccades, skips and regressions, can be used as features for NLP tasks (Mishra et al., 2018b). Since these gaze features relate to the cognitive process in reading (Altmann, 1994), we consider these as features in our model. The current gaze data extraction adds up all the interest areas on the screen (word + context). The software used to analyze the gaze data, currently, provides us with collated results for all the gaze-based features.

From the gaze behaviour data collected, we extract a total of 18 features for each of the 1800 data points. Using supervised feature selection approach, we are able to select eight best features via grid search using Logistic Regression. We use the SelectKBest implementation along with hyperparameter tuning via GridSearchCV, present in the sklearn (Pedregosa et al., 2011) library. Here onwards, we refer to these eight features when discussing cognitive or gaze-based features in this paper. These eight best features, along with their description, are listed in Table 4.

\subsection{Gaze Feature Prediction}

Collection of gaze data for a large number of samples can be a costly task. We propose a neural model for cognitive features prediction. Our neural model is a feed-forward neural network to perform a regression task and predict gaze features. We collect gaze data for only 200 word-pairs (D2) with the help of 9 annotators which provides us with a total of 1800 data points for training and validation. As reported in Table 5, the initial results on D1 using different cross-lingual embeddings show that XLM based contextual features perform the best amongst all the cross-lingual models.

As an input to the network, we provide the feature vectors from the XLM model. This network's output is the predicted gaze features for the D1 dataset, using the gaze features as gold predictions 


\begin{tabular}{cc}
\hline Gaze Feature & Description \\
\hline Average Fixation Duration & The average of all fixation duration across all interest areas present on the screen. \\
Average Saccade Amplitude & Saccade amplitude is the amplitude of going back and forth measured in terms of duration. \\
Fixation Count & Counting the number of times user's eyes are fixated on the screen. \\
Fixation Duration Max & Maximum time for a single fixation on any Interest Area. \\
Fixation Duration Min & Minimum time for a single fixation on any Interest Area. \\
IA Count & Interest Area Count (no. of IAs on the screen) \\
Run Count & Consecutive counts for same Interest Area are ignored in Run Count \\
Saccade Count & Total counts of Saccades \\
\hline
\end{tabular}

Table 4: Gaze Features used for the task of Cognate Detection

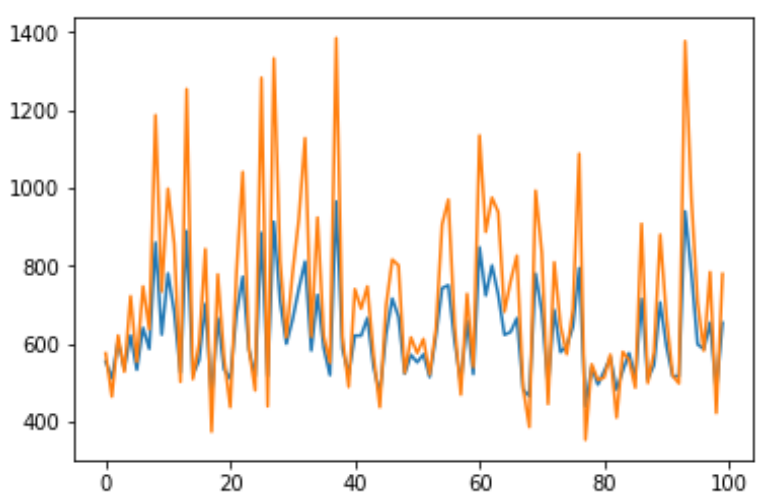

Figure 2: Predicted feature values ( blue ) vs. Gold feature values ( orange ) for the average fixation duration feature, on 100 samples.

from the D2 dataset. This network contains three linear hidden layers with 128, 64 and 32 dimensions. After each layer, we use the sigmoid activation function and dropout after each sigmoid with a dropout value of 0.2 . We use 0.1 as the learning rate and use the Mean Squared Error (MSE) loss function. A graph comparing the values for the predictions vs the actual values for the average saccade amplitude for 100 samples, can be seen in Figure 2.

\section{The Cognate Detection Task}

We employ both classical machine learning-based models and a simple feed-forward neural network. To compare our work with the previously proposed approaches, we replicate the best-reported systems from Rama (2016) i.e., Siamese Convolutional Neural Network with phonetic vectors as features and also replicate Kanojia et al. (2019b)'s approach which uses a Recurrent Neural Network architecture with a weighted lexical similarity (WLS) as the feature set. The input to our classifiers is the feature sets described above for each candidate pair.

Among the classical machine learning models, we use Support Vector Machines (SVM) and Logistic Regression (LR). We experiment with the use of both linear SVMs and kernel SVMs (Gaussian and
Polynomial). We perform a grid-search to find the best hyper-parameter value for $C$ over the range of 0.01 to 1000 . We deploy the Feed Forward Neural Network (FFNN) with one hidden layer. We perform cross-validation with different activation function settings (tanh, hardtanh, sigmoid and relu) and the hidden layer dimension in the network (30, 50,100 , and 150). We use binary cross-entropy as the optimization algorithm. Finally, we choose the hyper-parameter configuration with the best validation accuracy. We train the model with the selected configuration with an initial learning rate of 0.4 , and we halve the learning rate when the error on the validation split increases. We stop the training once the learning rate falls below 0.001. We perform 5-fold stratified cross-validation, which divides the data into train and test folds, randomly.

Initially, we perform our experiments with the feature sets from three different cross-lingual embeddings (MUSE, XLM, and VecMap) for the dataset D1, then with the smaller dataset D2 and later on the combined dataset D1+D2. We, then, perform the same task for the smaller dataset D2 by combining cognitive features with individual cross-lingual feature sets. We also observe the performance of standalone gaze features for the D2 dataset. Finally, we evaluate the predicted gaze features on the combined dataset by combining them with cross-lingual features and a standalone feature set, using the feed-forward neural network. We report the results of the cognate detection task in the next section and discuss them in detail.

\section{Results and Dicussion}

We report the results of the cognate detection task in Table 5. We use the original implementations of Rama (2016) and Kanojia et al. (2019b) on the combined (D1+D2) dataset. In our initial evaluation on the D1 dataset, cross-lingual model-based features (XLM, MUSE, and VecMap) can be seen to outperform the baseline systems which use phonetic and orthographic features. Using the XLM-based 


\begin{tabular}{|c|c|c|c|c|c|c|c|c|c|c|c|c|}
\hline & $\mathrm{P}$ & $\mathrm{R}$ & $\mathrm{F}$ & $\mathrm{P}$ & $\mathrm{R}$ & $\mathrm{F}$ & $\mathrm{P}$ & $\mathrm{R}$ & $\mathrm{F}$ & $\mathrm{P}$ & $\mathrm{R}$ & $\mathrm{F}$ \\
\hline Feature Set $\rightarrow$ & \multicolumn{3}{|c|}{ Phonetic } & \multicolumn{3}{|c|}{ WLS } & & & & & & \\
\hline Rama et. al., 2016 (D1+D2) & 0.71 & 0.69 & 0.70 & - & - & - & & & & & & \\
\hline Kanojia et. al., 2019 (D1+D2) & - & - & - & 0.76 & 0.72 & 0.74 & & & & & & \\
\hline Feature Set $\rightarrow$ & \multicolumn{3}{|c|}{ XLM } & \multicolumn{3}{|c|}{ MUSE } & \multicolumn{3}{|c|}{ VecMap } & & & \\
\hline Linear SVM (D1+D2) & 0.83 & 0.71 & 0.77 & 0.72 & 0.68 & 0.70 & 0.70 & 0.65 & 0.67 & & & \\
\hline LogisticRegression (D1+D2) & 0.85 & 0.74 & 0.79 & 0.80 & 0.71 & 0.75 & 0.70 & 0.66 & 0.68 & & & \\
\hline FFNN (D1 + D2) & 0.82 & 0.84 & 0.83 & 0.83 & 0.79 & 0.81 & 0.75 & 0.76 & 0.75 & & & \\
\hline Feature Set $\rightarrow$ & \multicolumn{3}{|c|}{ XLM+Gaze } & \multicolumn{3}{|c|}{ MUSE+Gaze } & \multicolumn{3}{|c|}{ VecMap+Gaze } & \multicolumn{3}{|c|}{ Gaze } \\
\hline Linear SVM (D2) & 0.81 & 0.69 & 0.75 & 0.72 & 0.73 & 0.72 & 0.70 & 0.75 & 0.72 & 0.77 & 0.76 & 0.76 \\
\hline LogisticRegression (D2) & 0.84 & 0.75 & 0.79 & 0.76 & 0.72 & 0.74 & 0.81 & 0.71 & 0.76 & 0.80 & 0.75 & 0.77 \\
\hline FFNN (D2) & 0.83 & 0.85 & 0.84 & 0.83 & 0.78 & 0.80 & 0.86 & 0.83 & 0.84 & 0.81 & 0.71 & 0.76 \\
\hline \multicolumn{13}{|c|}{ Predicted Gaze Features On D1 (11652 samples) and Collected Gaze Features on D2 (200 samples) } \\
\hline Feature Set $\rightarrow$ & \multicolumn{3}{|c|}{ XLM+Gaze } & \multicolumn{3}{|c|}{ MUSE+Gaze } & \multicolumn{3}{|c|}{ VecMap+Gaze } & \multicolumn{3}{|c|}{ Gaze } \\
\hline FFNN (D1 + D2) & 0.84 & 0.88 & 0.86 & 0.85 & 0.78 & 0.81 & 0.83 & 0.85 & 0.84 & 0.77 & 0.76 & 0.76 \\
\hline FFNN (D1) [Only Predicted Gaze] & 0.83 & 0.84 & 0.83 & 0.82 & 0.79 & 0.80 & 0.80 & 0.86 & 0.83 & 0.76 & 0.77 & 0.76 \\
\hline
\end{tabular}

Table 5: Classification results in terms of weighted Precision (P), Recall (R), and F-scores (F) using 5-fold crossvalidation using different feature sets as described above.

features, we observe an improvement of $9 \%$ over the stronger baseline (Kanojia et al., 2019b) and $13 \%$ over the system by Rama (2016). It can be seen that MUSE and VecMap based features also perform better on the combined dataset. In terms of both precision and recall, cross-lingual features are shown to outperform the baseline systems. The cross-lingual approach, with representations from VecMap-based model, fails to perform as well as MUSE and XLM-based models. The contextual XLM model achieves the best scores in almost all the settings. We believe its performance can be attributed to the linguistic closeness of the language pair and context from the contextual clues provided. For example, the false friend pair " $k a c c H a$ " (meaning inexperienced) - "kaccHa" [raw (food)] is classified correctly by XLM but incorrectly by both the baseline models, and VecMap-based classification. This signifies that fine-grained semantic difference between such false friend pairs can be captured via cognitive features. We report the additional results on individual datasets (D1 and D2), for all the baseline and cross-lingual approaches, in Table 6 .

For all the classifiers, the gaze features are averaged across participants and augmented with crosslingual features. The gaze fixation duration collects the total time spent, as fixations, on each interest area including the context clues. We were hopeful that the participants would focus only on important contextual clues and not the stop words with our experiment design. However, the sample points are not enough to concretely discuss this aspect of our study. These results are reported for all the classifiers with D2 dataset. Our feature combinations outperform the baselines with an F-score improvement of $10 \%$ points over the stronger baseline (WLS). We also report the precision, recall and F-score values when only gaze features are used to predict the labels for our candidate pairs. We observe that standalone gaze features are not as effective as when combined with cross-lingual feature vectors. When gaze features are predicted using the methodology described in Section 4.5, the model performance for FFNN on D1 remains the same with XLM+Gaze, decreases slightly for MUSE+Gaze and significantly improves for VecMap+Gaze. We observe that predicted gaze features do not significantly drop the performance, and hence, add the collected gaze data samples (D2) to D1.

On the combined dataset with collected gaze features (on D2) and predicted gaze features (on D1), we report our best system [FFNN (D1+D2)] which shown an improvement of $12 \%$ over the stronger baseline (WLS), and $16 \%$ over the weaker baseline (Phonetic). This system also outperforms the best reported cross-lingual features-based approach by $3 \%$, as shown in Table 5 .

For example, the cognate pair "utPaNa" (Hindi) "utpAaDit" (Marathi) (both meaning manufactured) is classified correctly by this system, but incorrectly 


\begin{tabular}{cccccccccc}
\hline & \multicolumn{3}{c}{ Phonetic } & \multicolumn{1}{c}{ WLS } & & & \\
\hline & P & R & F & P & R & F & P & R & F \\
\hline Rama et. al., 2016 (D1) & 0.70 & 0.68 & 0.69 & - & - & - & & & \\
Kanojia et. al., 2019 (D1) & - & - & - & 0.74 & 0.70 & 0.72 & & & \\
\hline Rama et. al., 2016 (D2) & 0.64 & 0.57 & 0.60 & - & - & - & & & \\
Kanojia et. al., 2019 (D2) & - & - & - & 0.61 & 0.66 & 0.63 & & & \\
\hline & & XLM & & & MUSE & & & VecMap & \\
\hline Linear SVM (D1) & 0.81 & 0.71 & 0.76 & 0.70 & 0.68 & 0.69 & 0.70 & 0.65 & 0.67 \\
LogisticRegression (D1) & 0.80 & 0.75 & 0.77 & 0.72 & 0.74 & 0.73 & 0.70 & 0.73 & 0.71 \\
FFNN (D1) & 0.80 & 0.84 & 0.82 & 0.81 & 0.76 & 0.78 & 0.77 & 0.76 & 0.76 \\
\hline Linear SVM (D2) & 0.72 & 0.65 & 0.68 & 0.65 & 0.60 & 0.62 & 0.62 & 0.57 & 0.59 \\
LogisticRegression (D2) & 0.78 & 0.69 & 0.73 & 0.67 & 0.67 & 0.67 & 0.63 & 0.61 & 0.62 \\
FFNN (D2) & 0.79 & 0.81 & 0.80 & 0.76 & 0.71 & 0.73 & 0.74 & 0.71 & 0.72 \\
\hline
\end{tabular}

Table 6: Additional results in terms of weighted Precision (P), Recall (R), and F-scores (F) using 5-fold crossvalidation using different feature sets as described above. These are additional results on the individual datasets D1 and D2 for which the combined results (D1 and D2) are already shown in Table 5 for a fair comparison.

by both the baselines, and all the cross-lingual systems. Furthermore, we also show that gaze features can be predicted based on a small sample data, and improved performance can be attained with the help of cross-lingual features, reported with our work.

\section{Conclusion and Future Work}

In this paper, we harness cross-lingual embeddings and gaze-based features to improve the task of cognate detection for the Indian language pair of HindiMarathi. We create a novel framework that derives insights from human cognition, that manifests over eye movement patterns. We hypothesize that by augmenting cross-lingual features with features obtained from the gaze data, the task of cognate detection can be improved. We use a linked knowledge graph (IndoWordnet) to augment a publicly released cognate dataset with contextual clues. We collect the gaze behaviour data from nine participants over 200 samples and perform the task of cognate detection for both our datasets (with gaze data and without gaze data). Then, we use a neural network to predict gaze features for unseen samples and perform the task of cognate detection to show improved performance, despite a small sample of collected gaze data.

We reproduce the previously proposed baseline approaches and perform experiments using additional features obtained via cross-lingual models for a comparative evaluation. The previously proposed approaches (Rama, 2016; Kanojia et al., 2019b) for this task are shown to be outperformed by cross-lingual features and the combination of these features with the obtained gaze data. Our experiments use three different approaches to generate feature representations for the cognate detection task, and all of them show improvements over previously proposed approaches. We observe consistent improvements in terms of precision, recall and F-scores. Over the stronger baseline, our best system shows an improvement of $12 \%$ points and $16 \%$ points over the weaker baseline. This system also outperforms the cross-lingual features based approaches by $3 \%$, over the combined dataset. We release this augmented dataset, along with our code and cross-lingual models for further research.

In future, we aim to add more language pairs and leverage contextual information from knowledge graphs using sequence-based neural models. We also aim to collect gaze data and then model the gaze predictions in a multi-task setting. We plan to investigate other multilingual contextual embeddings' performance for this task (e.g., M-BERT, IndicBERT, MuRIL). We also plan to look for a method to differentiate between different interest areas and see if a markup facility is present in the software used to analyze the gaze data. We also aim to investigate the task of cognate detection for the Indo-European language family, in the near future. 


\section{References}

Yaser Al-Onaizan, Jan Curin, Michael Jahr, Kevin Knight, John Lafferty, Dan Melamed, Franz-Josef Och, David Purdy, Noah A Smith, and David Yarowsky. 1999. Statistical machine translation. In Final Report, JHU Summer Workshop, volume 30.

Gerry TM Altmann. 1994. Regression-contingent analyses of eye movements during sentence processing: Reply to rayner and sereno. Memory \& Cognition, 22(3):286-290.

Mikel Artetxe, Gorka Labaka, and Eneko Agirre. 2017. Learning bilingual word embeddings with (almost) no bilingual data. In Proceedings of the 55th Annual Meeting of the Association for Computational Linguistics (Volume 1: Long Papers), pages 451-462, Vancouver, Canada. Association for Computational Linguistics.

Maria Barrett, Joachim Bingel, Nora Hollenstein, Marek Rei, and Anders Søgaard. 2018. Sequence classification with human attention. In Proceedings of the 22nd Conference on Computational Natural Language Learning, pages 302-312, Brussels, Belgium. Association for Computational Linguistics.

Maria Barrett, Joachim Bingel, Frank Keller, and Anders Søgaard. 2016. Weakly supervised part-ofspeech tagging using eye-tracking data. In Proceedings of the 54th Annual Meeting of the Association for Computational Linguistics (Volume 2: Short Papers), pages 579-584, Berlin, Germany. Association for Computational Linguistics.

Aditya Bhargava and Grzegorz Kondrak. 2009. Multiple word alignment with profile hidden markov models. In Proceedings of Human Language Technologies: The 2009 Annual Conference of the North American Chapter of the Association for Computational Linguistics, Companion Volume: Student Research Workshop and Doctoral Consortium, pages 43-48. Association for Computational Linguistics.

Pushpak Bhattacharyya. 2017. Indowordnet. In The WordNet in Indian Languages, pages 1-18. Springer.

Henrike K Blumenfeld and Viorica Marian. 2005. Covert bilingual language activation through cognate word processing: An eye-tracking study. In Proceedings of the Annual Meeting of the Cognitive Science Society, volume 27.

Evelyn Bosma and Naomi Nota. 2020. Cognate facilitation in frisian-dutch bilingual children's sentence reading: An eye-tracking study. Journal of experimental child psychology, 189:104699.

Alina Maria Ciobanu and Liviu P Dinu. 2014. Automatic detection of cognates using orthographic alignment. In Proceedings of the 52nd Annual Meeting of the Association for Computational Linguistics (Volume 2: Short Papers), volume 2, pages 99-105.
Alina Maria Ciobanu and Liviu P Dinu. 2015. Automatic discrimination between cognates and borrowings. In Proceedings of the 53rd Annual Meeting of the Association for Computational Linguistics and the 7th International Joint Conference on Natural Language Processing (Volume 2: Short Papers), volume 2, pages 431-437.

Alexis Conneau, Guillaume Lample, Marc'Aurelio Ranzato, Ludovic Denoyer, and Hervé Jégou. 2017. Word translation without parallel data. arXiv preprint arXiv:1710.04087.

Johannes Dellert. 2018. Combining informationweighted sequence alignment and sound correspondence models for improved cognate detection. In Proceedings of the 27th international conference on computational linguistics, pages 3123-3133.

Yerai Doval, Jose Camacho-Collados, Luis EspinosaAnke, and Steven Schockaert. 2018. Improving cross-lingual word embeddings by meeting in the middle. arXiv preprint arXiv:1808.08780.

Ana V González-Garduño and Anders Søgaard. 2018. Learning to predict readability using eye-movement data from natives and learners. In Thirty-Second AAAI Conference on Artificial Intelligence.

Bradley Hauer and Grzegorz Kondrak. 2011. Clustering semantically equivalent words into cognate sets in multilingual lists. In Proceedings of 5th international joint conference on natural language processing, pages 865-873.

Nora Hollenstein and Ce Zhang. 2019. Entity recognition at first sight: Improving NER with eye movement information. In Proceedings of the 2019 Conference of the North American Chapter of the Association for Computational Linguistics: Human Language Technologies, Volume 1 (Long and Short Papers), pages 1-10, Minneapolis, Minnesota. Association for Computational Linguistics.

Kenneth Holmqvist, Marcus Nyström, Richard Andersson, Richard Dewhurst, Halszka Jarodzka, and Joost Van de Weijer. 2011. Eye tracking: A comprehensive guide to methods and measures. OUP Oxford.

Gerhard Jäger, Johann-Mattis List, and Pavel Sofroniev. 2017. Using support vector machines and state-ofthe-art algorithms for phonetic alignment to identify cognates in multi-lingual wordlists. In Proceedings of the 15th Conference of the European Chapter of the Association for Computational Linguistics: Volume 1, Long Papers, volume 1, pages 1205-1216.

Marcel A Just and Patricia A Carpenter. 1980. A theory of reading: From eye fixations to comprehension. Psychological review, 87(4):329.

Diptesh Kanojia, Malhar Kulkarni, Pushpak Bhattacharyya, and Gholamreza Haffari. 2020. Challenge dataset of cognates and false friend pairs from indian languages. In Proceedings of The 12th Language Resources and Evaluation Conference, pages 3096-3102. 
Diptesh Kanojia, Malhar Kulkarni, Pushpak Bhattacharyya, and Gholemreza Haffari. 2019a. Cognate identification to improve phylogenetic trees for indian languages. In Proceedings of the ACM India Joint International Conference on Data Science and Management of Data, pages 297-300. ACM.

Diptesh Kanojia, Kevin Patel, Pushpak Bhattacharyya, Malhar Kulkarni, and Gholemreza Haffari. 2019b. Utilizing wordnets for cognate detection among indian languages. In Global Wordnet Conference (2019).

Sigrid Klerke, Yoav Goldberg, and Anders Søgaard. 2016. Improving sentence compression by learning to predict gaze. In Proceedings of the 2016 Conference of the North American Chapter of the Association for Computational Linguistics: Human Language Technologies, pages 1528-1533, San Diego, California. Association for Computational Linguistics.

Teuvo Kohonen. 1978. A very fast associative method for the recognition and correction of misspelt words, based on redundant hash addressing. In Proceedings of the fourth International Joint Conference on Pattern Recognition, 1978.

Grzegorz Kondrak. 2000. A new algorithm for the alignment of phonetic sequences. In Proceedings of the 1st North American chapter of the Association for Computational Linguistics conference, pages 288-295. Association for Computational Linguistics.

Grzegorz Kondrak. 2001. Identifying cognates by phonetic and semantic similarity. In Second Meeting of the North American Chapter of the Association for Computational Linguistics.

Saurav Kumar, Saunack Kumar, Diptesh Kanojia, and Pushpak Bhattacharyya. 2020. "a passage to India": Pre-trained word embeddings for Indian languages. In Proceedings of the 1st Joint Workshop on Spoken Language Technologies for Under-resourced languages (SLTU) and Collaboration and Computing for Under-Resourced Languages (CCURL), pages 352-357, Marseille, France. European Language Resources association.

Anoop Kunchukuttan, Divyanshu Kakwani, Satish Golla, Avik Bhattacharyya, Mitesh M Khapra, Pratyush Kumar, et al. 2020. Ai4bharat-indicnlp corpus: Monolingual corpora and word embeddings for indic languages. arXiv preprint arXiv:2005.00085.

Guillaume Lample and Alexis Conneau. 2019. Crosslingual language model pretraining. Advances in Neural Information Processing Systems (NeurIPS).

Johann-Mattis List. 2012. Lexstat: Automatic detection of cognates in multilingual wordlists. In Proceedings of the EACL 2012 Joint Workshop of LINGVIS \& UNCLH, pages 117-125. Association for Computational Linguistics.
Yunfei Long, Rong Xiang, Qin Lu, Chu-Ren Huang, and Minglei Li. 2019. Improving attention model based on cognition grounded data for sentiment analysis. IEEE Transactions on Affective Computing.

Sandeep Mathias, Rudra Murthy, Diptesh Kanojia, Abhijit Mishra, and Pushpak Bhattacharyya. 2020. Happy are those who grade without seeing: A multitask learning approach to grade essays using gaze behaviour. arXiv preprint arXiv:2005.12078.

I Dan Melamed. 1999. Bitext maps and alignment via pattern recognition. Computational Linguistics, 25(1):107-130.

Helen M Meng, Wai-Kit Lo, Berlin Chen, and Karen Tang. 2001. Generating phonetic cognates to handle named entities in english-chinese cross-language spoken document retrieval. In IEEE Workshop on Automatic Speech Recognition and Understanding, 2001. ASRU'01., pages 311-314. IEEE.

Paola Merlo and Maria Andueza Rodriguez. 2019. Cross-lingual word embeddings and the structure of the human bilingual lexicon. In Proceedings of the 23rd Conference on Computational Natural Language Learning (CoNLL), pages 110-120, Hong Kong, China. Association for Computational Linguistics.

Michelle M Mielke, Rosebud O Roberts, Rodolfo Savica, Ruth Cha, Dina I Drubach, Teresa Christianson, Vernon S Pankratz, Yonas E Geda, Mary M Machulda, Robert J Ivnik, et al. 2012. Assessing the temporal relationship between cognition and gait: slow gait predicts cognitive decline in the mayo clinic study of aging. Journals of Gerontology Series A: Biomedical Sciences and Medical Sciences, 68(8):929-937.

Abhijit Mishra and Pushpak Bhattacharyya. 2018. Cognitively Inspired Natural Language Processing: An Investigation Based on Eye-tracking. Springer.

Abhijit Mishra, Diptesh Kanojia, Seema Nagar, Kuntal Dey, and Pushpak Bhattacharyya. 2016. Harnessing cognitive features for sarcasm detection. In Proceedings of the 54th Annual Meeting of the Association for Computational Linguistics (Volume 1: Long Papers), pages 1095-1104, Berlin, Germany. Association for Computational Linguistics.

Abhijit Mishra, Srikanth Tamilselvam, Riddhiman Dasgupta, Seema Nagar, and Kuntal Dey. 2018a. Cognition-cognizant sentiment analysis with multitask subjectivity summarization based on annotators' gaze behavior. In Thirty-Second AAAI Conference on Artificial Intelligence.

Pushkar Mishra, Marco Del Tredici, Helen Yannakoudakis, and Ekaterina Shutova. 2018b. Author profiling for abuse detection. In Proceedings of the 27th International Conference on Computational Linguistics, pages 1088-1098, Santa Fe, New Mexico, USA. Association for Computational Linguistics. 
Andrea Mulloni and Viktor Pekar. 2006. Automatic detection of orthographics cues for cognate recognition. In LREC, pages 2387-2390.

John Nerbonne and Wilbert Heeringa. 1997. Measuring dialect distance phonetically. In Computational Phonology: Third Meeting of the ACL Special Interest Group in Computational Phonology.

Olumide Owolabi and DR McGregor. 1988. Fast approximate string matching. Software: Practice and Experience, 18(4):387-393.

Fabian Pedregosa, Gaël Varoquaux, Alexandre Gramfort, Vincent Michel, Bertrand Thirion, Olivier Grisel, Mathieu Blondel, Peter Prettenhofer, Ron Weiss, Vincent Dubourg, et al. 2011. Scikit-learn: Machine learning in python. the Journal of machine Learning research, 12:2825-2830.

Taraka Rama. 2016. Siamese convolutional networks for cognate identification. In Proceedings of $\mathrm{COL}$ ING 2016, the 26th International Conference on Computational Linguistics: Technical Papers, pages 1018-1027.

Taraka Rama, Johann-Mattis List, Johannes Wahle, and Gerhard Jäger. 2018. Are automatic methods for cognate detection good enough for phylogenetic reconstruction in historical linguistics? arXiv preprint arXiv:1804.05416.

Morteza Rohanian. 2017. Multi-document summarization of Persian text using paragraph vectors. In Proceedings of the Student Research Workshop Associated with RANLP 2017, pages 35-40, Varna. INCOMA Ltd.

Rosa M Sáchez-Casas, José E García-Albea, and Christopher W Davis. 1992. Bilingual lexical processing: Exploring the cognate/non-cognate distinction. European Journal of Cognitive Psychology, 4(4):293-310.

Claude E Shannon. 1948. A mathematical theory of communication. The Bell system technical journal, 27(3):379-423.

Abhinav Deep Singh, Poojan Mehta, Samar Husain, and Rajkumar Rajakrishnan. 2016. Quantifying sentence complexity based on eye-tracking measures. In Proceedings of the Workshop on Computational Linguistics for Linguistic Complexity (CLALC), pages 202-212, Osaka, Japan. The COLING 2016 Organizing Committee.

Eva Van Assche, Wouter Duyck, Robert J Hartsuiker, and Kevin Diependaele. 2009. Does bilingualism change native-language reading? cognate effects in a sentence context. Psychological science, 20(8):923-927.

Victoria Yaneva, Le An Ha, Richard Evans, and Ruslan Mitkov. 2020. Classifying referential and non-referential it using gaze. arXiv preprint arXiv:2006.13327. 